\title{
Combined phacoemulsification and microinvasive glaucoma surgery in comparison to phacoemulsification alone for open angle glaucoma
}

\author{
Graham A. Lee $\mathbb{1}^{1,2,3} \cdot$ Ashley J. Porter ${ }^{1} \cdot$ Roslyn A. Vincent ${ }^{1} \cdot$ Johanna Makk $^{1} \cdot$ Stephen J. Vincent ${ }^{4}$
}

Received: 13 May 2018 / Revised: 19 March 2019 / Accepted: 22 April 2019 / Published online: 10 May 2019

(c) The Royal College of Ophthalmologists 2019

\begin{abstract}
Importance Microinvasive glaucoma surgery (MIGS) combined with phacoemulsification is increasingly utilised in glaucoma management.

Objective To describe the postoperative outcomes of phacoemulsification alone compared to combined phacoemulsification and iStent (Glaukos, San Clemente, CA) or Hydrus (Ivantis, Irvine, CA) for open angle glaucoma.

Methods Retrospective single surgeon comparative case series in a private practice setting. A total of 297 eyes in 190 patients $(\mathrm{M}: \mathrm{F}=85: 105)$ were included consecutively from March 2011 to June 2017 with the data analysed using linear mixed models. Main outcome measures were intraocular pressure (IOP) and number of medications.

Results By 6 months, all groups showed a reduction in IOP, with the combined iStent group by $4.2 \mathrm{~mm} \mathrm{Hg}$ and the combined Hydrus group by $4.5 \mathrm{~mm} \mathrm{Hg}$, and this trend was sustained with follow-up to two years. All groups also showed a reduction in number of medications, with the phacoemulsification alone cases by 0.3 , the combined iStent group by 0.7 and the combined Hydrus group by 1.1 medications. Both the combined surgeries had sustained reduction of number of medications with follow-up to 2 years. Postoperative complications from MIGS device insertion were uncommon and all resolved by one month.

Conclusions and Relevance The combination of phacoemulsification and a MIGS device in open angle glaucoma patients reduced the intraocular pressure and the number of medications by the first postoperative month and had longer-term effects at 2 years follow-up. If the device is inserted without intraoperative complication, the rate of significant short-term risks are low.
\end{abstract}

\section{Introduction}

The surgical management of open angle glaucoma (OAG) has progressed over the last 50 years. Since the earlier Scheie procedures with a scleral fistula, it has evolved into the guarded filtration techniques of trabeculectomy and glaucoma drainage devices [1]. More recently, microstents have been introduced to assist with improving the internal outflow of aqueous humour [1, 2]. Early evidence

Graham A. Lee

eye@cityeye.com.au

1 City Eye Centre, Brisbane, QLD, Australia

2 University of Queensland, Brisbane, QLD, Australia

3 Mater Health Services, Brisbane, QLD, Australia

4 Queensland University of Technology, Brisbane, QLD, Australia supporting the biofeasibility of the microstents in rabbits and non-human primates was conducted by Grierson et al. [3]. They demonstrated collector channel patency, associated with minimal granulation and fibrosis after microstent insertion [3]. The collector channels have been verified to remain patent and increase aqueous outflow by reducing resistance [3].

The main indication for microinvasive glaucoma surgery (MIGS) is in patients with mild to moderate OAG [4, 5]. These devices have been approved for standalone use or concurrently with cataract extraction. The current study investigates the early postoperative and long-term effects of two MIGS devices iStent (Glaukos, San Clemente, CA) and Hydrus (Ivantis, Irvine, CA) in combination with phacoemulsification in terms of change of intraocular pressure (IOP) and number of glaucoma medications. The results are compared to two separate control groups consisting of patients with and without OAG undergoing phacoemulsification. 


\section{Methods}

This is a retrospective consecutive case series of patients that presented to a subspecialty cataract and glaucoma private practice (GL) in Brisbane, Australia, between March 2011 and June 2017 for cataract surgery with or without MIGS insertion. There was a single point of care throughout the study including the surgery, postoperative visits and decisions on altering glaucoma medications. Institutional ethical approval for the trial was obtained through the University of Queensland (Number 2016-05-440). All study procedures adhered to the Declaration of Helsinki for research involving humans and written informed consent was obtained from the study participants.

Patients were included in the review if they were undergoing phacoemulsification for cataract removal, and if they met the criteria for microstent insertion through having mild to moderate OAG (primary open-angle glaucoma, pseudoexfoliation glaucoma or pigmentary glaucoma) and did not require other glaucoma surgical management such as trabeculectomy or glaucoma drainage device. The patients were chosen consecutively, with the first group consisting of OAG patients undergoing phacoemulsification, the next group on introduction of the iStent then followed by the third group on introduction of the Hydrus. Data for the first postoperative month of nonglaucoma patients undergoing routine phacoemulsification were collected consecutively during the period of data collection. Patient data collected included age, gender, type of OAG, surgical operation undertaken, the pre- and postoperative IOP and number of medications up to two years post-surgery. The inraocular pressure (IOP) was mainly measured with the iCare rebound tonometer (iCare, Oy, Vantaa, Finland) by clinical assistants masked to the type of stent, however, the Goldman applanation tonometer was also used in some cases. The iCare rebound tonometer records 6 readings over a $2 \mathrm{~s}$ period, discarding the highest and lowest reading and averages the remaining 4 readings. This device has a high level of agreement with Goldman applanation tonometry in treated glaucoma patients. Where multiple IOP measurements had been recorded in the patient files, the highest value was included in the data collection. The measurements reflected real-life practice and there was no medication washout period. The glaucoma medications were adjusted according to the IOP at the observed time points and the target IOP required for that patient. Prostaglandin analogues were avoided in the first postoperative month, with either a topical carbonic anhydrase inhibitor or alpha-agonist or a combination with timolol $0.5 \%$ prescribed. After this early postoperative period, the patients requiring glaucoma medications were switched to either monotherapy with a prostaglandin analogue and medications later added according to the IOP response. All patients were prescribed topical antibiotic and steroids during the first postoperative month.

\section{Surgical technique}

Phacoemulsification was performed via a temporal clear corneal incision. If a MIGS device was to be inserted after intraocular lens insertion, the viscoelastic device was irrigated and aspirated from the anterior chamber, intracameral acetylcholine 1\% (Miochol, Alcon, Fortworth, USA) was injected to constrict the pupil and sodium hyaluronate $1.4 \%$ (Healon GV, AMO, Irvine, USA) used to fill the anterior chamber. A silicone gel pillow was placed under the patient's shoulder nearest to the surgeon and the patient's head and surgical microscope were rotated to create an angle of 60-80 degrees to allow optimum gonioscopic view of the angle. The trabecular meshwork was identified with the gonioprism and the MIGS device implanted. The viscoelastic device was irrigated and aspirated from the anterior chamber and replaced with a balanced salt solution. Intracameral antibiotic and subconjunctival steroid was injected unless contraindicated.

Linear mixed models were used to examine the magnitude of change in IOP and the number of glaucoma medications over time (preoperative and 6,12 and 24 months postoperative), between treatment groups (phacoemulsification alone, combined iStent and combined Hydrus) and their interaction (time by treatment group). A first-order autoregressive repeated covariance type was used which assumes that adjacent measurements from the same individual are more highly correlated than observations farther apart in time. Given the known high correlation between fellow eyes for numerous variables, whether data was included from one or both eyes of an individual was also incorporated in the models as a fixed factor (however this was not a statistically significant factor). $P$ values of $<0.05$ were considered statistically significant and a Bonferroni correction was applied to adjust for multiple comparisons. The data are presented as the mean or mean difference and the standard error of the mean (with 95\% confidence intervals). Frequencies were examined using Chi-Square analysis.

\section{Results}

A total of 297 eyes in 190 patients (M:F, 85:105) with a mean age of $72.1 \pm 10$ years (range 32-99 years) were included in this study (Table 1). Half of the patients' eyes did not have OAG $(n=148)$ while the OAG patients were evenly divided between the phacoemulsification alone $(n=$ $47)$, combined iStent $(n=50)$ and combined Hydrus $(n=$ 
Table 1 Baseline characteristics of the study groups (\%)

\begin{tabular}{lllll}
\hline & $\begin{array}{l}\text { Phacoemulsification without } \\
\text { OAG } n=148\end{array}$ & $\begin{array}{l}\text { Phacoemulsification with OAG } \\
n=47\end{array}$ & Combined iStent $n=50$ & Combined Hydrus $n=52$ \\
Sex M:F & $77(52.0): 71(48.0)$ & $24(51.1): 23(48.9)$ & $15(30.0): 35(70.0)$ & $19(36.5): 33(63.5)$ \\
Primary OAG & & $41(87.2)$ & $50(96.2)$ & $49(94.2)$ \\
$\begin{array}{l}\text { Pseudoexfoliation } \\
\text { glaucoma }\end{array}$ & & $5(10.6)$ & $2(3.9)$ & $3(5.8)$ \\
Pigmentary glaucoma & & $1(2.1)$ & $0(0)$ & $0(0)$ \\
Age & $70 \pm 12$ & $73 \pm 9$ & $70 \pm 8$ & $70 \pm 9$ \\
IOP (mmHg) & 14.7 & 13.6 & 17.9 & 16.8 \\
& $(95 \%$ CI 13.3-16.2) & $(95 \%$ CI $11.3-16.0)$ & $(95 \%$ CI 15.2-20.6) & $(95 \%$ CI $14.3-19.4)$ \\
Meds & 0.0 & 2.1 & 2.6 & 2.6 \\
& & $(95 \%$ CI 1.8-2.4) & $(95 \%$ CI 2.3-3.0) \\
\hline
\end{tabular}

52) groups. Preoperatively, the mean IOPs were in those without OAG $14.7 \mathrm{mmHg}$ (95\% CI 13.3-16.2); OAG and phacoemulsification alone $13.6 \mathrm{mmHg} \quad(95 \% \quad \mathrm{CI}$, 11.3-16.0); combined iStent $17.9 \mathrm{mmHg} \quad(95 \% \mathrm{CI}$, 15.2-20.6); and combined Hydrus $16.8 \mathrm{mmHg}$ (95\% CI, 14.3-19.4). The mean number of medications used were in those without OAG 0.0 ; OAG and phacoemulsification alone 2.1 (95\% CI, 1.8-2.4); combined iStent 2.6 (CI, 2.2-3.0); and combined Hydrus 2.6 (95\% CI, 2.3-3.0) (Table 1). All OAG patients completed at least 6 months follow-up and up to 24 months follow-up. For some patients, the 6 months data was not available, but were reviewed later at 12 and/or 24 months. The final sample size for the OAG patients included in the statistical analysis was 149 (preoperative), 140 (94.0\%) (6 months), 140 (94.0\%) (12 months) and 126 (84.6\%) (24 months), representing 6-15\% missing data across the follow-up visits. The sample size for each treatment group at each visit is included in Figs. 1 and 2.

\section{Intraocular pressure}

\section{Short term}

A significant treatment group by time interaction was observed. On the first postoperative day, the phacoemulsification alone groups recorded a rise in IOP; those without OAG $(n=148)$ by $2.6 \mathrm{mmHg}$ [95\% CI, 1.8 to $3.5 \mathrm{~mm} \mathrm{Hg}$ ] while those with OAG $(n=47)$ by $4.8 \mathrm{mmHg}$ [95\% CI, 3.3 to $6.3 \mathrm{mmHg}$ ]. The combined iStent group $(n=50)$ reduced IOP by $3.0 \mathrm{mmHg}$ [95\% CI, -4.4 to $-1.6 \mathrm{~mm} \mathrm{Hg} ; p<$ $0.0001]$ and the combined Hydrus group $(n=52)$ by 5.9 $\mathrm{mm} \mathrm{Hg}$ [95\% CI, -7.3 to $-4.5 \mathrm{~mm} \mathrm{Hg} ; p<0.0001]$. At 1 month, the phacoemulsification alone cases without OAG and with OAG both decreased the IOP by $2.1 \mathrm{mmHg}$ [95\% $\mathrm{CI},-3.1$ to $-1.1 \mathrm{~mm} \mathrm{Hg}$ ] and $1.1 \mathrm{~mm} \mathrm{Hg}$ [95\% CI, -2.8 to $0.6 \mathrm{~mm} \mathrm{Hg}$ ], respectively. The combined iStent group showed a reduction by $2.0 \mathrm{mmHg}$ [95\% CI, -3.5 to -0.4
$\mathrm{mmHg}$ ), and the combined Hydrus group by $3.5 \mathrm{~mm} \mathrm{Hg}$ [95\% CI, -4.9 to $-2.0 \mathrm{~mm} \mathrm{Hg}$ ].

\section{Long term}

Follow-up of the OAG groups at 6, 12, and 24 months showed a stable IOP for the iStent group (13.7, $n=46$; $14.2, n=46 ; 13.8, n=46)$, Hydrus $(12.3, n=50 ; 12.5, n$ $=49 ; 12.6, n=39)$ and was better controlled than phacoemulsification alone $(12.2, n=44 ; 12.4, n=45 ; 12.6, n$ $=41$ ) respectively (Figure 1 ). The combined iStent and Hydrus groups displayed a similar reduction in IOP across all postoperative follow-up visits (all visits, $\mathrm{p}>0.05$ ). Posthoc comparisons revealed that the IOP reduction was significantly greater in both MIGS groups compared to the phacoemulsification alone group at the 6 months (both $p<$ 0.01), 12 months (both $p<0.01$ ) and 24 months follow-up visits (both $p<0.05$ ). The initial reduction in IOP observed at 6 months remained stable at 24 months across each treatment group $(p>0.05)$.

\section{Glaucoma medications}

The number of medications in the OAG patients at baseline was similar between the groups ranging from 2.1 to 2.6 (Table 1) with most patients using a combination of a prostaglandin analogue with beta-blocker, alpha-agonist and/or topical carbonic anhydrase inhibitor. The number of medications required at six months was reduced in the phacoemulsification alone with OAG group $(0.3 \pm 0.1$ less medications [95\% CI, 0.0 to 0.5$]$ ), the combined iStent group $(0.7 \pm 0.2$ less medications [95\% CI, 0.3 to 1.0$])$ and in the combined Hydrus group $(1.2 \pm 0.1$ less medications [95\% CI, 0.9 to 1.5$]$ ) (all $p<0.001$ compared to preoperative) (Figure 2). The reduction in the number of medications was greater in both MIGS groups compared to phacoemulsification alone ( $p<0.05$ at all visits), with the combined Hydrus group displaying a greater reduction in 
Fig. 1 Boxplots of the change in intraocular pressure in patients undergoing phacoemulsification with and without microstent insertion (showing 95\% confidence intervals)

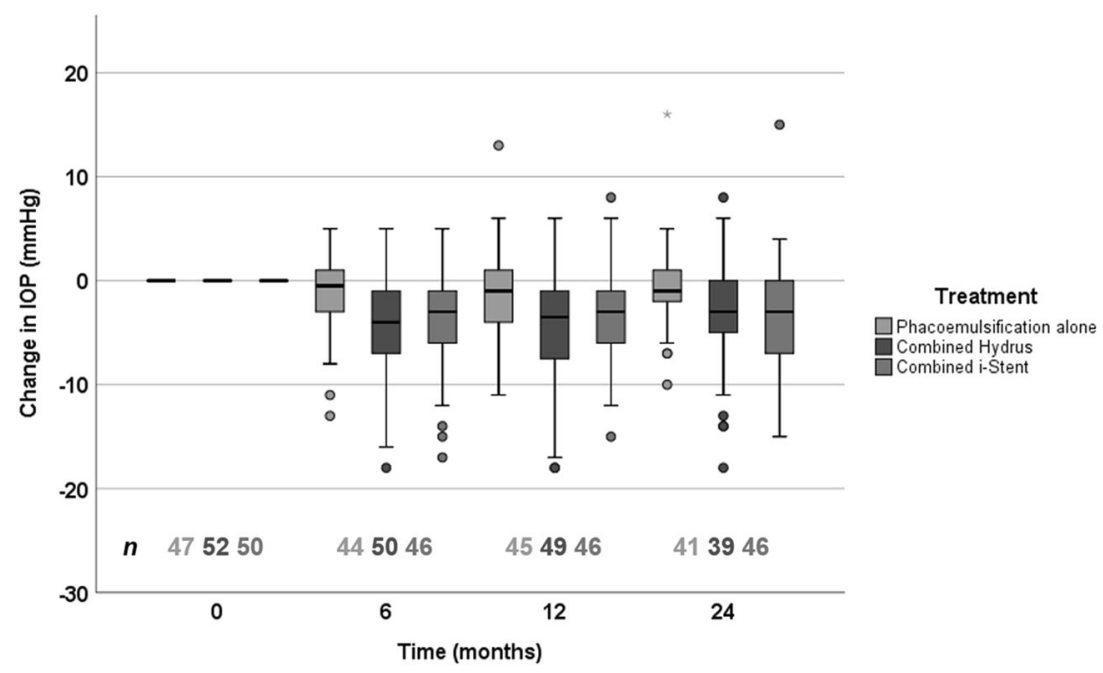

Fig. 2 Boxplots of the change in number of medications in patients undergoing phacoemulsification with and without microstent insertion (showing 95\% confidence intervals)

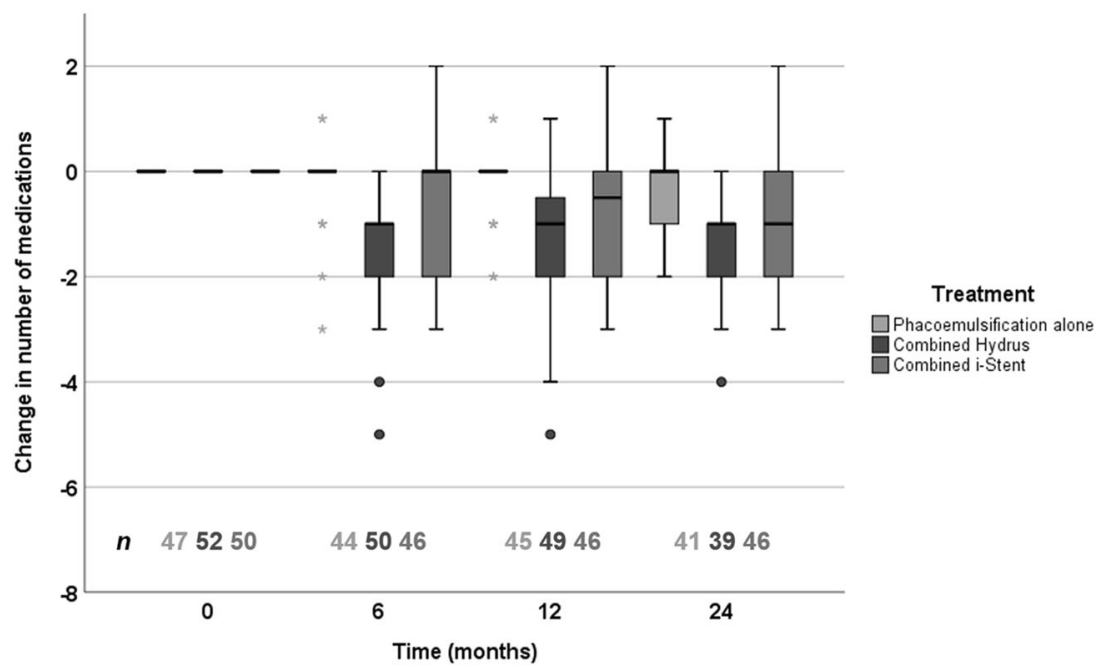

medications at the 6 months visit compared to the combined iStent group $(0.5 \pm 0.2$ less medications, $95 \%$ CI 0.1 to 0.9$)$ $(p=0.03)$. The reduction in the number of medications remained stable up to 24 months.

There were a significantly greater number of patients who were medication free by 12 months, in the combined iStent group $13(28.3 \%)$ and the combined Hydrus group 14 $(28.6 \%)$ in comparison to the phacoemulsification group with OAG $2(4.4 \%)$ (both $p<0.01)$.

\section{Complications}

In five eyes, the iStent (3) and Hydrus stents (2) were unable to be placed due to inadequate view from bleeding (cases excluded from the data). One patient who received a Hydrus stent developed a hyphema of more than $1.0 \mathrm{~mm}$ in the early postoperative period and resolved spontaneously without sequelae (Figures 3a, b). In nine eyes, Hydrus repositioning was performed intraoperatively, with the initial temporal approach unsuccessful and subsequent superior approach insertion. In one eye, the microstent was partially dislocated outside Schlemm's canal postoperatively (Fig. 3c). One iStent patient required trabeculectomy as the IOP was not adequately controlled. There were no adverse visual outcomes associated with combining phacoemulsification with MIGS insertion such as increased incidence of cystoid macula oedema, corneal decompensation or significant refractive error.

In patients without OAG, 12 eyes $(8.1 \%)$ had an IOP spike $>30 \mathrm{mmHg}$ on the first postoperative day, while with OAG, 8 eyes $(17.0 \%)$ had the same IOP spike. In the combined iStent group, two eyes $(3.9 \%)$ had an IOP spike on day one, while only one patient $(1.9 \%)$ in the combined Hydrus group recorded an IOP spike. No eyes developed flat anterior chambers or choroidal effusions and all cases of low pressure were transient, settling spontaneously within the one month postoperative period. Long-term complications included peripheral 
Fig. 3 a Day one hyphema of $>1 \mathrm{~mm}$, with blot clot at pupil margin. b Slit lamp view of hyphema. c Gonioscopic view of Hydrus stent showing partial dislocation from trabecular meshwork

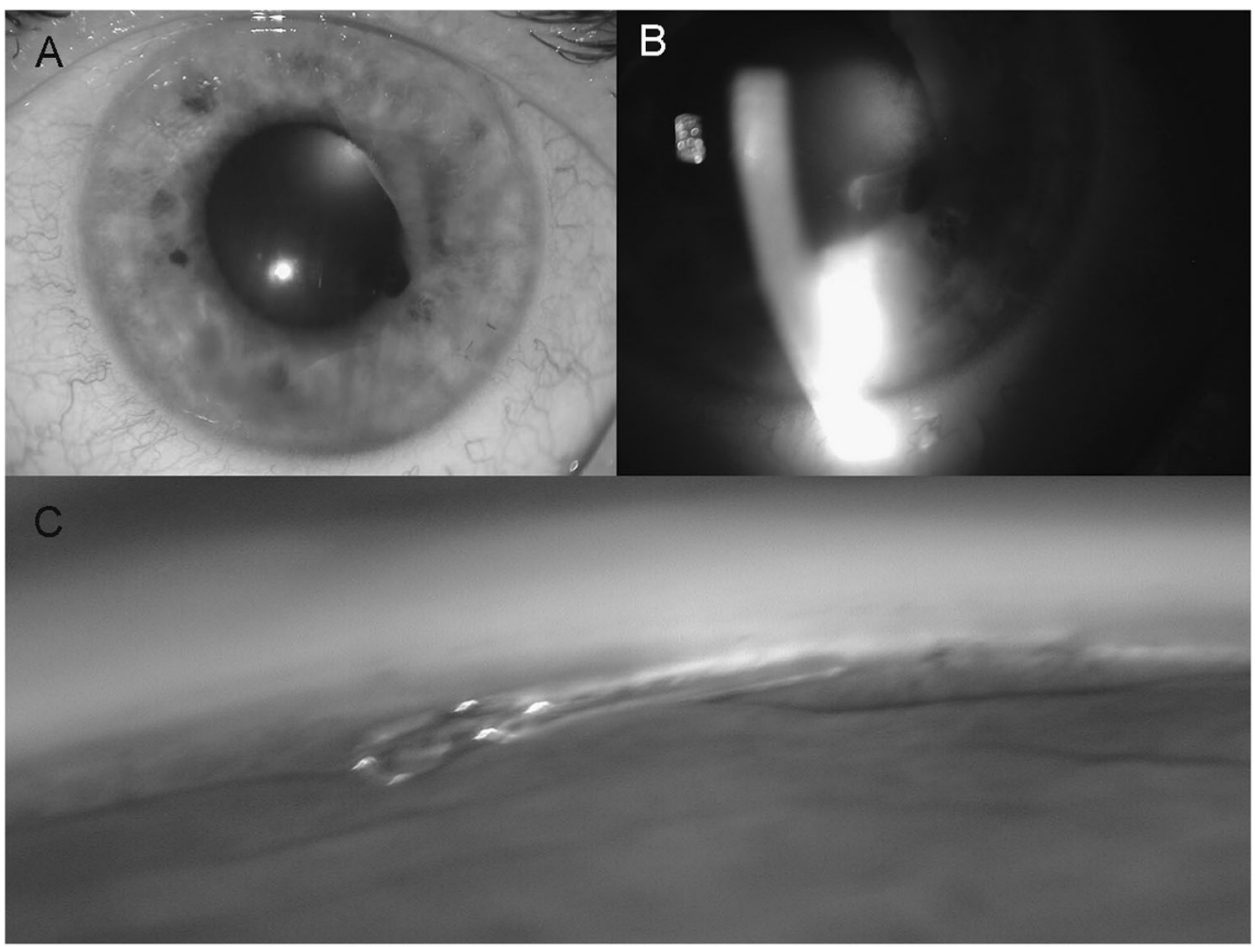

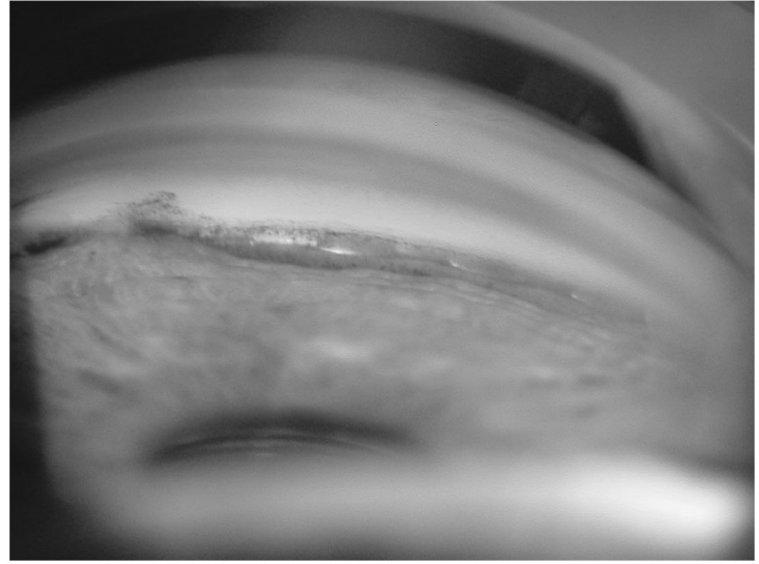

Fig. 4 Gonioscopic view of Hydrus stent implanted for 3 years, showing peripheral anterior synechiae to opening and fibrosis to overlying trabecular meshwork

anterior synechiae to the stent ostium and fibrosis of the trabecular meshwork in both the iStent and the Hydrus stent (Figure 4), however in this case the IOP was 10 $\mathrm{mmHg}$ on no medications.

\section{Discussion}

This study compares the relative efficacy of two MIGS devices with phacoemulsification alone using real world data from a single surgeon case series. The early postoperative period has not been previously welldocumented as most reports have addressed longer-term results. MIGS has been described as minimally invasive surgery, however, there are potential complications as outlined in this study. For all new devices, there is a learning curve, so documentation of the early operative period can be beneficial to less experienced surgeons. Intraoperative technique, for example correct stent placement, minimal collateral damage to surrounding structures, thorough viscoelastic device removal and control of bleeding from the stent can all affect the early IOP levels. By six months, the IOP and number of medications remained relatively stable in those cases followed for up to two years.

The control of the IOP following phacoemulsification can be a challenge in glaucoma. Glaucoma patients have a tendency for pressure spikes in the postoperative period that may be visually threatening, particularly in advanced optic neuropathy [6]. The efficacy of phacoemulsification at lowering IOP in patients with OAG is reported to be $1.4-2.0 \mathrm{~mm} \mathrm{Hg}[7,8]$. The efficacy of the iStent has been reported. Fea et al. showed a reduction in IOP by $3.2 \mathrm{~mm} \mathrm{Hg}$ with $67 \%$ medication free at 15 months [4]. Samuelson et al. found that at 12 months $72 \%$ achieved an IOP $\leq 21 \mathrm{~mm} \mathrm{Hg}$ without medication and $66 \%$ had an IOP reduction $>20 \%$ [5]. This same group followed the patients at 24 months and recorded that $61 \%$ had achieved an IOP $\leq 21 \mathrm{mmHg}$ without medication and that $53 \%$ had an IOP reduction $>20 \%$ at 24 months [9]. In comparison, Pfeiffer et al. investigated phacoemulsification and concurrent Hydrus insertion with 
phacoemulsification alone and found $80 \%$ of those in the Hydrus group had a $20 \%$ reduction in diurnal IOP at 24 months and $73 \%$ did not require any glaucoma medications [10]. Another study by Fea et al. reported that the Hydrus combined with phacoemulsification showed an IOP reduction of $3.7 \mathrm{mmHg}$ and $64 \%$ of patients were medication free at 2 years [11]. In this particular study, the proportion of patients that were medication free was lower, as there was no set target IOP for the groups and clinically combining the medication and MIGS effects, achieved lower target IOP for the individual patient.

We found a statistically significant difference between the iStent and the Hydrus microstent in preventing the postoperative day one pressure spike when compared with phacoemulsification alone in open angle glaucoma patients. This may relate to the initial increase in the outflow facility by opening of Schlemms canal by the $9 \mathrm{~mm}$ long Hydrus compared to the smaller $1 \mathrm{~mm}$ long iStent. This initial difference in IOP lowering effects between the stents was present at one month however the longer-term follow-up did not show significant differences. Both microstents showed a reduction in the number of medications compared with phacoemulsification alone, however the Hydrus showed a 0.5 medication reduction advantage over the iStent ( $p=0.03$ ). This may be clinically relevant over many years with potentially less eyedrop usage and reduced ocular surface toxicity.

MIGS is associated with complications specific to the device insertion. Fea et al. reported one patient with hyphema of $>2.0 \mathrm{~mm}$ that resolved spontaneously, similar to the patient in our study [11]. Microscopic hyphema occurs following MIGS procedure due to the insertion into the trabecular meshwork, but tends to clear quickly with the increased trabecular outflow. If the IOP is less than the episcleral venous pressure, reflux of blood will occur via the stent into the anterior chamber. Intraoperative repositioning of the stent either due to wrong location of the stent or inadequate operating field of view can occur, requiring intraoperative explantation and insertion in another position for example, moving from a temporal to a superior approach. This can occur during the learning curve but also with more experienced surgeons, due to variations in ocular anatomy. Hypotony has not previously been reported with the current study's MIGS devices, however could potentially occur due to a localised cyclodialysis from traumatic stent insertion. No cases have so far required explantation of the stent or cleft repair. Malposition of the stent if not rectified intraoperatively, could result in damage to adjacent structures, however corneal decompensation, iris trauma, uveitis, hyphema, pigment dispersion have seldom been reported. Later complications include iris tissue adhesion to the stent and has occurred with both the iStent and Hydrus devices [10-12]. These studies have shown no adverse effect on visual outcome associated with combined phacoemulsification and MIGS [5, 9-12].

This study has examined the effect of MIGS in the early postoperative period, particularly in regards to the blunting of IOP spikes. This is of importance in advanced glaucoma patients and may have a protective role to augment other operations that increase IOP levels including insertion of glaucoma drainage devices, penetrating keratoplasties and vitrectomy operations. MIGS case selection is important and is not indicated for more rapidly progressing glaucoma patients who preferably require trabeculectomy or tube insertion. Further studies will elucidate the role of MIGS in the overall glaucoma management. Carefully guided training for all new surgeons is paramount for any procedure involving the delicate structures of the angle.

\section{Summary}

\section{What was known before}

- MIGS devices have been shown to decrease IOP and the number of medications in early to moderate open angle glaucoma.

- MIGS has a good safety profile, but varies depending on the type of device used.

\section{What this study adds}

- MIGS combined with phacoemulsification in open angle glaucoma patients shows a blunting of IOP spikes on the first postoperative day.

- Direct comparison of combined iStent and combined Hydrus groups in this study, showed similar IOP and number of medication lowering with a follow-up of up to two years.

- Postoperative complications from iStent and Hydrus devices are related to intraoperative insertion and resolved by one month.

Funding The Hydrus stent was supplied by Ivantis, Irvine, CA.

\section{Compliance with ethical standards}

Conflict of interest The authors declare that they have no conflict of interest.

Publisher's note: Springer Nature remains neutral with regard to jurisdictional claims in published maps and institutional affiliations. 


\section{References}

1. Razeghinejad MR, Spaeth GL. A history of the surgical management of glaucoma. [Review]. Optom Vis Sci. 2011;88: E39-47.

2. Freedman J. What is new after 40 years of glaucoma implants. J Glaucoma. 2010;19:504-8.

3. Grierson I, Saheb H, Kahook MY, Johnstone MA, Ahmed II, et al. A novel Schlemm's Canal scaffold: histologic observations. J Glaucoma. 2015;24:460-8.

4. Fea AM. Phacoemulsification versus phacoemulsification with micro-bypass stent implantation in primary open-angle glaucoma: randomized double-masked clinical trial. J Cataract Refract Surg. 2010;36:407-12.

5. Samuelson TW, Katz LJ, Wells JM, Duh YJ, Giamporcaro JE. Randomized evaluation of the trabecular micro-bypass stent with phacoemulsification in patients with glaucoma and cataract. Ophthalmology. 2011;118:459-67.

6. Tranos P, Bhar G, Little B. Postoperative intraocular pressure spikes: the need to treat. Eye. 2004;18:673-9.

7. Shingleton BJ, Pasternack JJ, Hung JW, O'Donoghue MW. Three and five year changes in intraocular pressures after clear corneal phacoemulsification in open angle glaucoma patients, glaucoma suspects, and normal patients. J Glaucoma. 2006;15:494-8.

8. Thomas R, Walland M, Thomas A, Mengersen K. Lowering of intraocular pressure after phacoemulsification in primary openangle and angle-closure glaucoma: a bayesian analysis. Asia Pac. J Ophthalmol. 2016;5:79-84.

9. Craven ER, Katz LJ, Wells JM, Giamporcaro JE. Cataract surgery with trabecular micro-bypass stent implantation in patients with mild-to-moderate open-angle glaucoma and cataract: two-year follow-up. J Cataract Refract Surg. 2012;38:1339-45.

10. Pfeiffer N, Garcia-Feijoo J, Martinez-de-la-Casa JM, Larrosa JM, Fea A, Lemij H, et al. A randomized trial of a schlemm's canal microstent with phacoemulsification for reducing intraocular pressure in open-angle glaucoma. Ophthalmology. 2015;122:1283-93.

11. Spiegel D, Wetzel W, Neuhann T, Stuermer J, Hoeh H, GarciaFeijoo J, et al. Coexistent primary open-angle glaucoma and cataract: interim analysis of a trabecular micro-bypass stent and concurrent cataract surgery. Eur J Ophthalmol. 2009;19:393-9.

12. Fea AM, Rekas M, Au L. Evaluation of a Schlemm canal scaffold microstent combined with phacoemulsification in routine clinical practice: Two-year multicenter study. J Cataract Refract Surg. 2017;43:886-91. 